\title{
Pänomen Bonding
}

\section{Einfluss des Bindungsverhaltens zwischen Mutter und Kind auf das Ernährungsverhalten}

Das Phänomen Bonding hat in den vergangenen Jahren stark an Bedeutung gewonnen. Dabei geht es um den frühen Beziehungsaufbau zwischen Mutter/Vater und dem Neugeborenen. Immer mehr werden sich Pflegekräfte, Ärzte und natürlich auch Eltern bewusst, welche grundlegende Basis für alle weiteren Entwicklungen durch ein gelungenes Bonding gegeben wird. Dies gilt für sämtliche kognitiven Bereiche wie auch soziales Verhalten. Dazu zählt unter anderem auch die Ernährungssituation bzw. das Ernährungsverhalten.

Bereits 1936 erforschte Bowlby in England den Wert der Bindungsförderung und bis in die Gegenwart unterstützen Forschungsarbeiten diese Untersuchungen. Eines ist vor allem wichtig zu betonen: Bindung kann niemals als statisches Geschehen betrachtet werden, sondern vielmehr ist es ein ununterbrochener Prozess.

Bereits intrauterin steigen Mutter und Kind in einen ununterbrochen Bindungsprozess ein. Dieser ist semibewusst und hat auch Auswirkungen auf den Hormonhaushalt. Postpartum setzt sich dieser Prozess ohne Unterbrechung fort: hormonell wie auch psychisch; so bewirkt eine hohe Oxytocinausschüttung neben verstärkten Uteruskontraktionen eine erhöhte Lernbereitschaft, Reduzierung von Ängstlichkeit und Stress wie auch die Bereitstellung des Kolostrums.

Alleine diese angeführten Faktoren machen klar, warum Bindungsaufbau wichtig war und ist: er sicherte das Überleben der Nachkommen durch beständiges Pflege- und Fütterungsverhalten der Eltern, machte alle Involvierten neugierig und lembereit füreinander und last but not least - wirkt sich unwillkürlich auf das Ernährungsverhalten aus.

Diese Phänomene waren nicht nur in Urzeiten wichtig, sondern sind es auch jetzt in unsere durchstrukturierten, scheinbar kalkulierten Zeit.

Wir kennen alle die Fragen die so gut wie alle Mütter von Anfang an beschäftigen:

- Kann ich/mag ich stillen?

Dr. Maria Weissenböck, Klinische und Gesundheitspsychologin, Psychotherapeutin, Supervisorin, Mediatorin, Coach, Wien

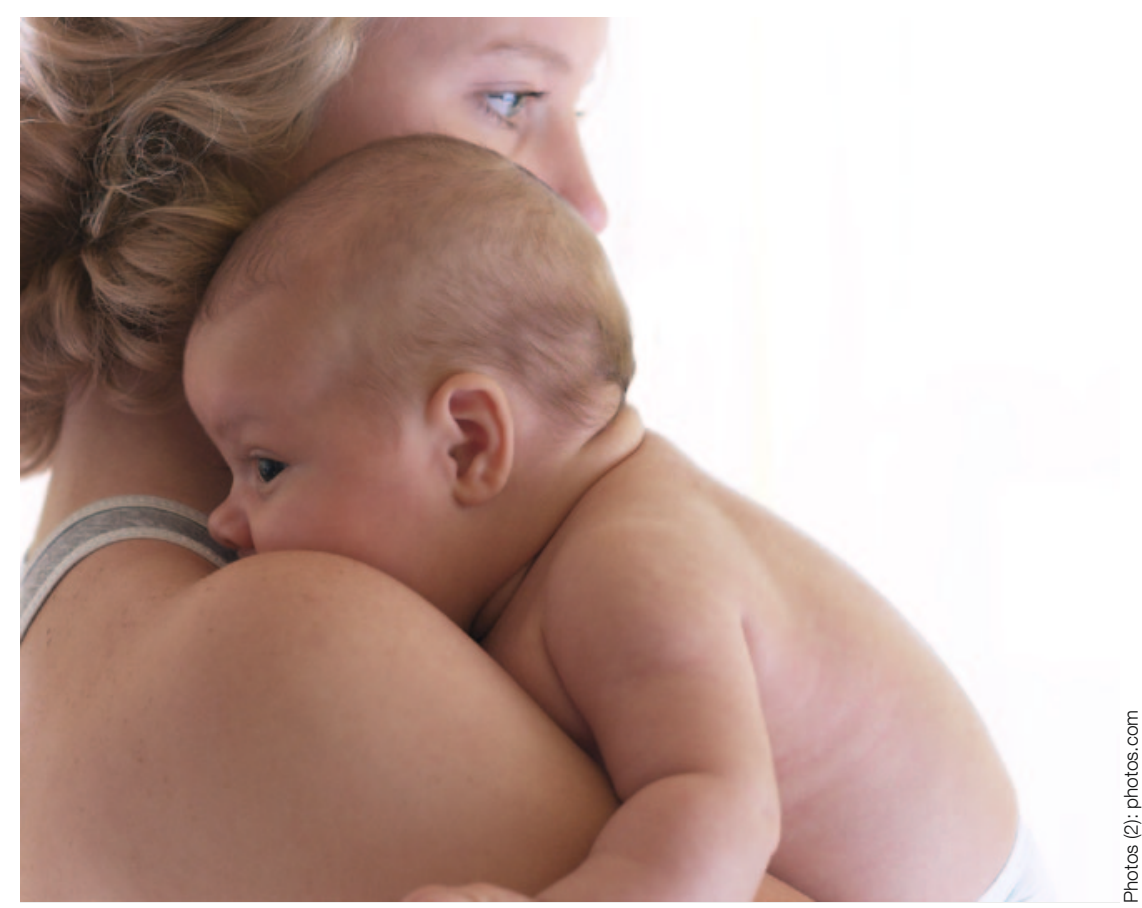

Bindung kann nie als statisches Geschehen betrachtet werden, sondern ist ein kontinuierlicher Prozess.

- Habe ich überhaupt genug Milch?

- Nimmt mein Baby genug zu?

Sobald es aus irgendwelchen Gründen zu „Fütter- und Gedeihstörungen“ kommt, kippt der kontinuierliche Prozess: es kommt zu einer Destabilisierung beider Seiten. Gekennzeichnet ist diese durch exzessives Schreien, Schlafstörungen, Interaktionsprobleme, Verunsicherung, Ängste und Schuldgefühle... um nur einige wenige aufzuzählen.

Ein beinahe unaufhaltsamer Teufelkreis dysfunktionaler Interaktionen entsteht. $\mathrm{Zu}$ durchbrechen gelingt dieser dann, wenn es zu Reflexionen der Mutter/ Bezugsperson kommt:

- Welche eigenen Esserfahrungen bringt diese mit?

- Welchen Stellenwert hat Nahrungsaufnahme generell in ihrem Leben?

- Welche Rückmeldungen seitens des Kindes gibt es und werden diese wahrgenommen?

Daneben ist es von Vorteil Eltern zu ermutigen die Bedürfnis-Sprache des Kindes wahrzunehmen, zu interpretieren und angemessen/prompt mit passendem/spie-

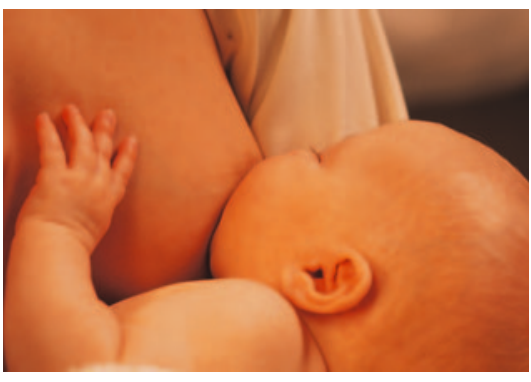

Bindungsaufbau sichert das Überleben der Nachkommen durch beständiges Pflege- und Fütterverhalten der Eltern.

gelndem Verhalten auf das des Kindes zu reagieren.

Daraus ergibt sich von selbst die Wechselwirkung von Bonding und Ernährungsverhaltens

Korrespondenz:

Dr. Maria Weissenböck

Gregor-Mendel-Str. 37

1190 Wien

E-Mail: maria@weissenboeck.at

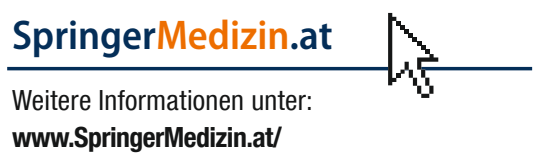

